\title{
Metamaterial-Based Wireless RF-MEMS Strain Sensors
}

\author{
$\underline{\text { Rohat Melik }}{ }^{1}$, Emre Unal ${ }^{1}$, Nihan Kosku Perkgoz ${ }^{1}$, Christian Puttlitz ${ }^{2}$, and Hilmi Volkan Demir ${ }^{1}$ \\ ${ }^{1}$ Department of Electrical and Electronics Engineering, Department of Physics, UNAM - National Nanotechnology Research \\ Center, and Institute of Materials Science and Nanotechnology, Bilkent University, Ankara, 06800, Turkey, E-mail address: \\ rohat@ee.bilkent.edu.tr; tel: +90 312290 2305; fax: + 90312290 1015; \\ ${ }^{2}$ Department of Mechanical Engineering, Colorado State University, Fort Collins, CO 80523, USA
}

\begin{abstract}
Approximately $10 \%$ of the fractures do not heal properly because of the inability to monitor fracture healing. Standard radiography is not capable of discriminating whether bone healing is occurring normally or aberrantly. We propose and develop an implantable wireless sensor that monitors strain on implanted hardware in real time telemetrically. This enables clinicians to monitor fracture healing. Here we present the development and demonstration of metamaterial-based radiofrequency (RF) micro-electro-mechanical system (MEMS) strain sensors for wireless strain sensing to monitor fracture healing. The operating frequency of these sensors shifts under mechanical loading; this shift is related to the surface strain of the implantable test material. In this work, we implemented metamaterials in two different architectures as bio-implantable wireless strain sensors for the first time. These custom-design metamaterials exhibit better performance as sensors than traditional RF structures (e.g., spiral coils) because of their unique structural properties (splits). They feature a low enough operating frequency to avoid the background absorption of soft tissue and yield higher Q-factors compared to the spiral structures (because their gaps have much higher electric field density). In our first metamaterial architecture of an $5 \times 5$ array, the wireless sensor shows high sensitivity $(109 \mathrm{kHz} / \mathrm{kgf}$, $5.148 \mathrm{kHz} /$ microstrain) with low nonlinearity-error (<200microstrain). Using our second architecture, we then improved the structure of classical metamaterial and obtained nested metamaterials that incorporate multiple metamaterials in a compact nested structure and measured strain telemetrically at low operating frequencies. This novel nested metamaterial structure outperformed classical metamaterial structure as wireless strain sensors. By employing nested metamaterial architecture, the operating frequency is reduced from $529.8 \mathrm{MHz}$ to $506.2 \mathrm{MHz}$ while the sensitivity is increased from $0.72 \mathrm{kHz} / \mathrm{kgf}$ to $1.09 \mathrm{kHz} / \mathrm{kgf}$.
\end{abstract}

Keywords-metamaterial; split ring resonator; nested metamaterials; remote sensing; strain; sensitivity; resonance frequency; RF-MEMS.

\section{INTRODUCTION}

Approximately six million long bone fractures are reported per annum in the USA. Surprisingly, approximately $10 \%$ of these fractures do not heal properly because of the inability to monitor fracture healing [1], [2]. For that reason, surgeons would like to asses fracture healing in real time through monitoring strain telemetrically. This requires an implantable wireless sensor. For operation of such a sensor, when external load is applied to the implantable test material, the strain is

This work is supported by TÜBA-GEBIP, ESF-EURYI, NRF-RF and TÜBITTAK EEEAG 107E088, 109E002, 109E004, and 110E010, and EU N4E NoE. This work is also supported by a subcontract from the United States National Institutes of Health (NIH) 5R01EB010035. induced on the test material, the sensor on the test material is deformed under stress, the dielectric capacitance $\left(\mathrm{C}_{\text {diel }}\right)$ and capacitance between gaps $\left(\mathrm{C}_{\text {gap }}\right)$ change, and hence the operating frequency changes. We read strain telemetrically from this operating frequency shift.

There has been a large amount of research work reported on metamaterials and their application areas including negative refraction [3], cloaking [4], superlenses [5], and focusing light [6]. However, metamaterial architectures have not been studied for bioimplant sensing till date. We employ metamaterials as bioimplant wireless strain sensors for the first time because of the benefits of their unique structural properties in wireless strain sensing. In this paper, we also propose a novel architecture, nested metamaterials, which outperforms the classical metamaterials as wireless strain sensors.

\section{ClassiCAL METAMATERIAL}

To have good wireless strain sensors, four important criteria are required. Firstly, the sensor must have a low enough operating frequency (sub $\mathrm{GHz}$ range) to get rid of the background absorption problems of soft tissue. At high frequencies, we would not receive good signals because of the absorption coefficient of soft tissue and electromagnetic waves do not penetrate in soft tissue at higher frequencies. Reducing the operating frequency may be the most important problem for wireless strain sensors since the sensor size is limited by plate size and normal size of the antenna for radiation is $\lambda_{0} / 4$. Secondly, the sensors must have a high Q-factor in order to track the operating frequency and operating frequency shift easily. Thirdly, the sensor should have high sensitivity. Since there are a limited number of data points in one frequency scan of the network analyzer, it is easier to resolve smaller shifts in the transmission spectra with respect to the externally applied load when the sensitivity is higher. Finally, linearity of sensing is important since with low nonlinearity-errors, we would easily monitor strain by just looking at the slope of the shift of operating frequency vs. microstrain curve. This criterion is related to Q-factor since a sensor with high Q-factor will have higher signal to noise ratio (SNR) and will have less nonlinearity-errors.

Metamaterials are better wireless strain sensors compared to conventional RF structures such as spiral coils, because their unique structural properties give advantages over conventional structures considering the four criteria listed above. There is a huge electric field density localization between the gaps of 
metamaterials so they provide higher signal; hence they exhibit higher Q-factors and higher dips in their transmission spectra compared to conventional RF structures (e.g., spiral coils). This enables us to make telemetric measurements and observe the operating frequency relatively more easily. These gaps also produce additional capacitance $\left(\mathrm{C}_{\text {gap }}\right)$, thus they feature higher shift of operating frequency with the same applied load, leading to higher sensitivity. Additional capacitance also results in lower resonance frequency per unit area, which is important for better sensing in body to avoid the background absorption of soft tissue. Besides, metamaterials demonstrate better linearity since they yield higher Q-factors. Higher Qfactor yields higher signal to noise ratio (SNR), which results in better linearity. Therefore, because of the gaps in metamaterial structure, we obtain higher Q-factors, higher dips, higher sensitivities, better linearity, and lower resonance frequency per unit area compared to spiral coil structure.

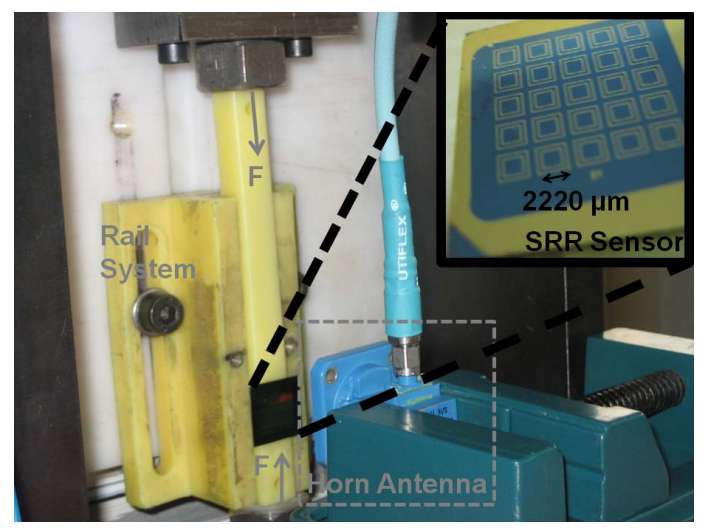

Figure 1. Our $5 \times 5$ metamaterial sensor in our compression set up.

We first deposit the $0.1 \mu \mathrm{m}$ thick $\mathrm{Si}_{3} \mathrm{~N}_{4}$ onto the silicon substrate, for the fabrication of our sensors, using plasma enhanced chemical vapor deposition (PECVD). Subsequently, by utilizing lithography, by making metallization using a boxcoater, and lift-off, we deposit and pattern a $0.1 \mu \mathrm{m}$ Au layer onto the $\mathrm{Si}_{3} \mathrm{~N}_{4}$ dielectric thin film and finalize our structure. Our final geometry is shown in Fig. 1 (denoted as SRR sensor) [1], with a $2220 \mu \mathrm{m}$ outer length and a $1500 \mu \mathrm{m}$ inner length. This structure also has an $80 \mu \mathrm{m}$ inner width and an $80 \mu \mathrm{m}$ outer width, with a $280 \mu \mathrm{m}$ inner spacing and a $280 \mu \mathrm{m}$ outer spacing, respectively. The unit cell length of one metamaterial structure is $2780 \mu \mathrm{m}$. We have a $5 \times 5$ array of these metamaterial unit cells incorporated in the sensor, resulting in a total of $1.5 \mathrm{~cm} \times 1.5 \mathrm{~cm}$ chip size. We fix our sensor to the test material via hard epoxy where we employ a cast polyamide stick as the test material. We use the custom-design compression setup for high frequency experiments, which applies compressive loads to the cast polyamide, stick from 0 kgf to $300 \mathrm{kgf}$ as shown in Fig. 1. We use standard gain horn antennas as external antennas where one antenna acts as the transmitter and the other as the receiver. For calibration purposes, we first measure the transmission of the implantable stick with no sensor chip attached. Subsequently, we repeat the same measurement with the sensor attached under no load and then, following application of discrete compressive loads. By this way, we obtain the transmission spectra referenced relative to the no sensor condition as a function of the applied load.

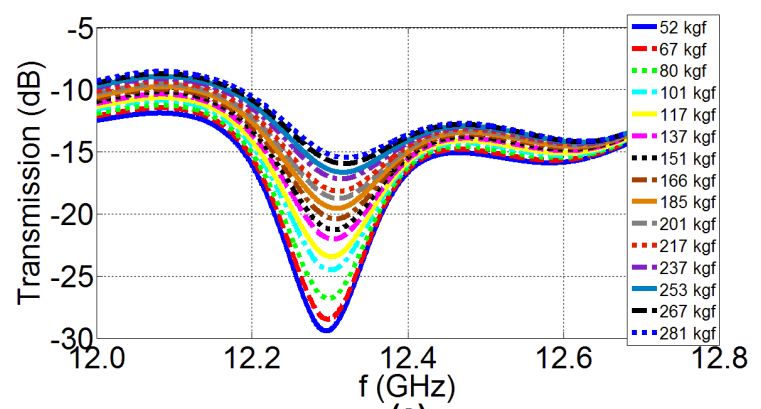

(a)
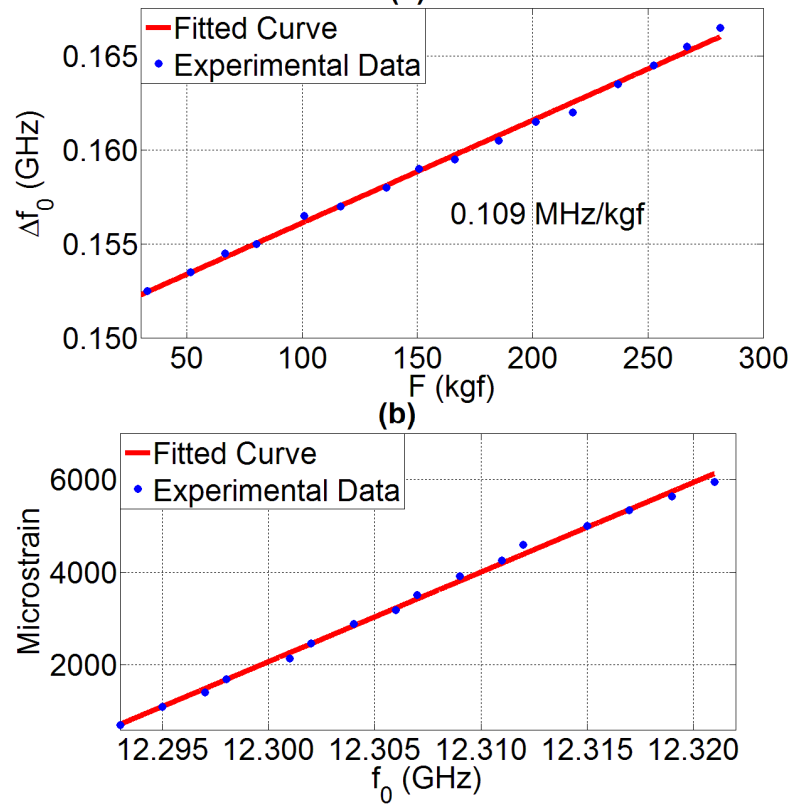

(c)

Figure 2. a) Transmission spectra of our metamaterial based wireless sensor parameterized with respect to the external force, b) its operating frequency shift as a function of applied force, and c) microstrain vs. operating frequency.

We can see the experimental results of classical metamaterial for high frequency experiments in Fig. 2. We can observe the transmission spectra of the sensor in Fig. 2(a), and from this curve, we can obtain $F$ vs. $\Delta$ fo (shift of operating frequency) in Fig. 2(b). We observe increase in operating frequency with applied load since in compression, the dielectric area and capacitance (dielectric capacitance) are decreased, the spacing between the metals is increased, and the capacitance between metals is decreased. Thus, the operating frequency increases. Also, since we know the young's modulus of the cast polyamide stick as $3.287 \mathrm{GPa}$, which is separately verified using a wired strain gauge (Tokyo Sokki Kenkyujo Co., Ltd. Strain Gauges with a gauge factor of 2.1), we extract the applied microstrain. Then by just looking at the shift of operating frequency, we can detect strain wirelessly as in Fig. 2(c). From the results, we demonstrate that our wireless sensors yield high sensitivity $(109 \mathrm{kHz} / \mathrm{kgf}, 5.148 \mathrm{kHz} /$ microstrain) with low nonlinearity-error ( $<200$ microstrain, $<6 \%$ ). These results indicate that metamaterials can be used in bioimplant sensing, 


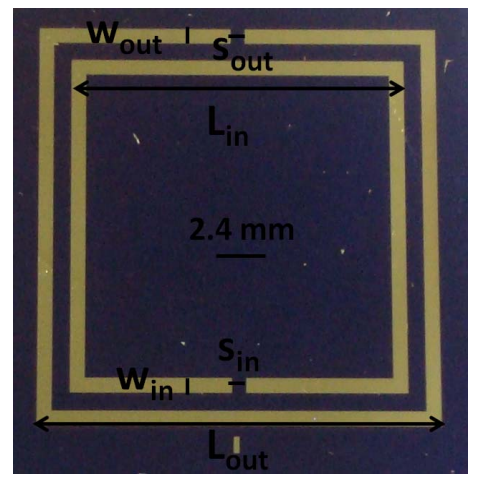

(a)

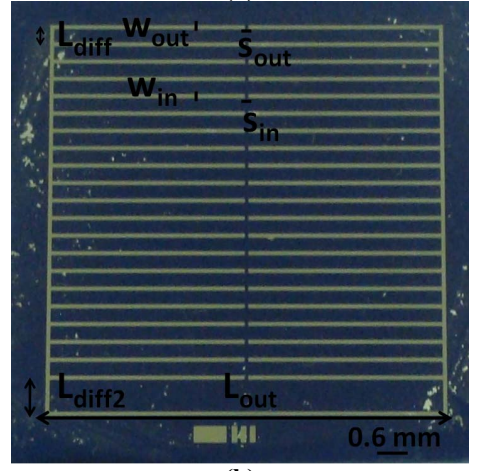

(b)

Figure 3. Plan view pictures of (a) the classical metamaterial structure and (b) our nested metamaterial structure.

which opened up possibly a new direction for metamaterials' applications.

\section{NESTED METAMATERIALS}

In our novel structure nested metamaterial [2], multiple metamaterials are nested together in one structure. It has more gaps compared to classical metamaterial structure hence it has additional capacitance compared to classical metamaterial, then it has lower operating frequency and higher sensitivity compared to classical metamaterial structure. Thus, it outperforms classical metamaterial in wireless strain sensing. The most important part of the nested metamaterial structure is its lowest coil. If we open a gap in the lowest coil, then we lose

Table I

The parameters of classical metamaterial

\begin{tabular}{|c|c|c|c|c|c|}
\hline $\begin{array}{c}\mathbf{L}_{\text {out }} \\
(\mathbf{m m})\end{array}$ & $\begin{array}{c}\mathbf{L}_{\text {in }} \\
(\mathbf{m m})\end{array}$ & $\begin{array}{c}\mathbf{w}_{\text {out }} \\
\mathbf{w}_{\text {in }} \\
(\mathbf{m m}\end{array}$ & $\begin{array}{c}\mathbf{S}_{\text {out }} \\
\mathbf{S}_{\text {in }} \\
(\mathbf{m m})\end{array}$ & $\begin{array}{c}\mathbf{t}_{\text {film }} \\
(\boldsymbol{\mu m})\end{array}$ & $\begin{array}{c}\mathbf{t}_{\text {metal }} \\
(\mu \mathbf{m})\end{array}$ \\
\hline $\mathbf{2 2 . 2}$ & $\mathbf{1 8 . 6}$ & $\mathbf{0 . 8}$ & $\mathbf{0 . 8}$ & $\mathbf{0 . 1}$ & $\mathbf{0 . 1}$ \\
\hline \multicolumn{6}{|c}{ Table II }
\end{tabular}

The parameters of nested metamaterial

\begin{tabular}{|c|c|c|c|c|c|c|c|}
\hline $\begin{array}{c}\text { The paramet } \\
(\mathbf{m m})\end{array}$ & $\begin{array}{c}\mathbf{W}_{\text {out }} \\
\mathbf{W}_{\text {in }} \\
(\mathbf{m m})\end{array}$ & $\begin{array}{c}\mathbf{s}_{\text {out }} \\
\mathbf{S}_{\text {in }} \\
(\mathbf{m m})\end{array}$ & $\begin{array}{c}\mathbf{L}_{\text {diff }} \\
(\mathbf{m m})\end{array}$ & $\begin{array}{c}\mathbf{L}_{\text {diff }} \\
(\mathbf{m m})\end{array}$ & $\begin{array}{c}\mathbf{t}_{\text {film }} \\
(\mu \mathbf{m})\end{array}$ & $\begin{array}{c}\mathbf{t}_{\text {metal }} \\
(\boldsymbol{\mu m})\end{array}$ & $\mathbf{N}$ \\
\hline $\mathbf{2 2 . 2}$ & $\mathbf{0 . 2}$ & $\mathbf{0 . 2}$ & $\mathbf{0 . 8}$ & $\mathbf{1 . 8}$ & $\mathbf{0 . 1}$ & $\mathbf{0 . 1}$ & $\mathbf{2 0}$ \\
\hline
\end{tabular}

the advantage of using multiple metamaterials nested together, then the operating frequency will increase. The classical metamaterial is two turns and we can increase the number of turns to decrease the operating frequency. However, this time, the parasitic capacitance dominates and we cannot preserve the Q-factor. Nevertheless, we can preserve the Q-factor while we decrease the operating frequency with nested metamaterial structure. Moreover, by increasing the number of turns in classical structure, we can decrease the operating frequency up to a point, but with our novel nested structure, by just playing with number of legs, width and spacing between gaps, we can easily adjust the operating frequency and decrease it.

In this section, as opposed to Section II, we make low frequency experiments. The structures used for low frequency experiments are fabricated in the same way as the structures used for high frequency experiments. We list the design parameters of classical and nested metamaterial structures in Table I, and Table II. We present the fabricated classical metamaterial structure and nested metamaterial structure in Fig. 3.

We experimentally characterize our sensors for low frequency experiments with the custom-design tension set up. As opposed to the compression set up, tension set up applies tensile force to cast polyamide stick, between 0-300 kgf. We use two coaxial probes as external antennas and observe the transmission of the sensor using network analyzer. We use coaxial probes, instead of using high-powered antennas such as standard gain horn antennas because of the size issues. Since classical radiating size is $\lambda_{\mathrm{o}} / 4$, the high-power antennas cannot see our devices at these sizes so we use coaxial probes with comparable sizes. Although these antennas are weak, since our devices have high Q-factors, we can take good data with these antennas. The calibration procedure used is the same procedure as we mentioned in high frequency experiments.

We show the transmission spectra of classical metamaterial in Fig. 4(a). The operating frequency of the classical metamaterial under no load is $529.8 \mathrm{MHz}$, thus the size of this classical metamaterial sensor is $\lambda_{\mathrm{o}} / 25.5$. From the transmission spectra with respect to different applied loads, we obtain F vs. $\Delta f_{o}$ (shift of operating frequency) with a sensitivity of 0.723 $\mathrm{kHz} / \mathrm{kgf}$ in Fig. 4(b) and acquire induced strain vs. $\Delta \mathrm{f}_{\mathrm{o}}$ in Fig. 4(c) with a sensitivity of $0.0259 \mathrm{kHz} /$ microstrain. As opposed to the compressive load, under tensile load, the gaps of metamaterials are decreased, hence $\mathrm{C}_{\text {gap }}$ is increased. Also the dielectric area between substrate and metal layer is increased, thus $\mathrm{C}_{\text {diel }}$ is increased. Therefore, the operating frequency decreases in tension as opposed to compression case. The sensor demonstrates $<500$ microstrain nonlinearity-error, which corresponds to $<16 \%$ nonlinearity-error.

From the transmission spectra of nested metamaterial sensor in Fig. 5(a), we can see that the operating frequency is decreased to $506.2 \mathrm{MHz}$ under no load, and the electrical size is also decreased to $\lambda_{0} / 26.7$. The sensitivities are increased to $1.09 \mathrm{kHz} / \mathrm{kgf}$ or $0.0369 \mathrm{kHz} /$ microstrain as shown in Fig. 5(b) and Fig. 5(c) respectively. The sensor exhibits $<600$ microstrain nonlinearity-error, corresponding to $<16 \%$ nonlinearity-error. The nonlinearity-errors of the nested metamaterial sensors are nearly the same as those of the classical nested metamaterial sensor while, the nested metamaterial sensor exhibits reduced operating frequency and increased sensitivity. 


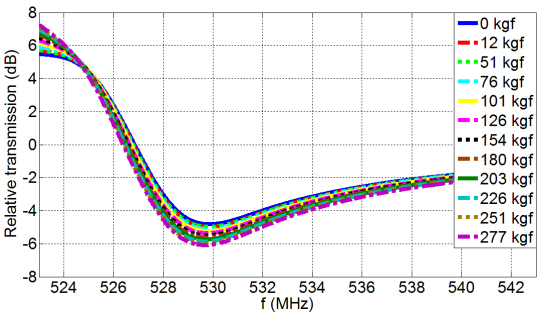

(a)

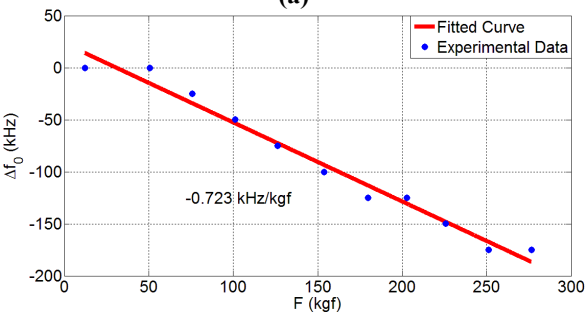

(b)

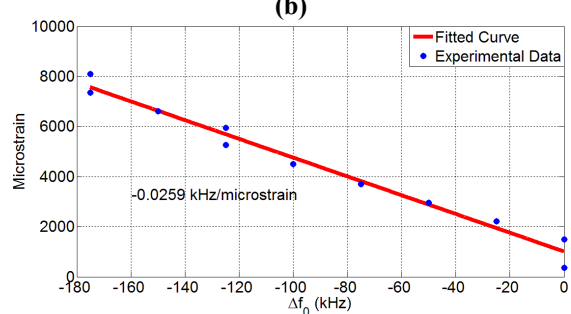

(c)

Figure 4. Experimental characterization of the classical metamaterial sensor under tension: (a) relative transmission spectra, (b) frequency shift $\left(\Delta \mathrm{f}_{\mathrm{o}}\right)$ vs. applied load $(\mathrm{F}),(\mathrm{c})$ induced strain (microstrain) vs. frequency $\operatorname{shift}\left(\Delta \mathrm{f}_{\mathrm{o}}\right)$.

\section{CONCLUSION}

In conclusion, we designed and implemented metamaterials as implantable wireless RF-MEMS strain sensors for the first time. By using metamaterials, we can obtain higher Q-factors, higher transmission dips, higher operating frequency shifts, high sensitivities, and better linearity compared to conventional RF structures such as spiral coils because of their unique structural properties (splits). Our metamaterial sensors yield high sensitivity $(109 \mathrm{kHz} / \mathrm{kgf}, 5.148 \mathrm{kHz} /$ microstrain) with low nonlinearity-error $(<200$ microstrain, $<6 \%)$. These results show that metamaterials can be used in bioimplant sensing, which opens up possibly a new direction for metamaterial applications. After high frequency experiments, we discovered a novel structure called nested metamaterials by increasing the number of splits and incorporating multiple metamaterials nested together in one structure. We compared classical metamaterial and nested metamaterial in low frequency experiments. The nested metamaterial structure outperformed classical metamaterial structure as wireless sensors. They have reduced operating frequency and increased sensitivity because they have much more gaps compared to classical metamaterial. The no-load operating frequency of nested metamaterial (506.2 $\mathrm{MHz}$ ) was decreased relative to the classical metamaterial

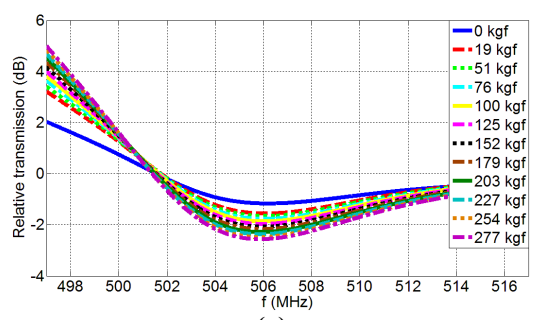

(a)

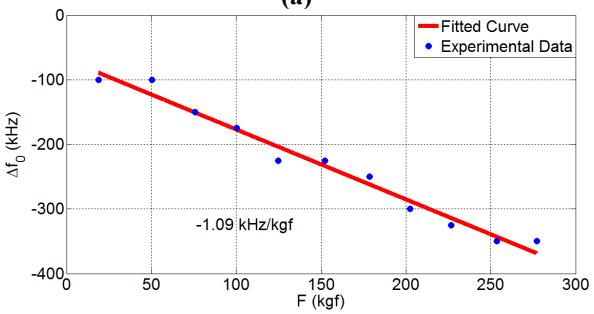

(b)

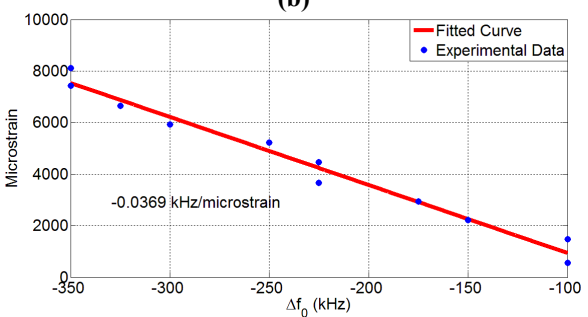

(c)

Figure 5. Experimental characterization of the nested metamaterial sensor under tension: (a) relative transmission spectra, (b) frequency shift $\left(\Delta \mathrm{f}_{\mathrm{o}}\right)$ vs. applied load $(\mathrm{F}),(\mathrm{b})$ induced strain (microstrain) vs. frequency $\operatorname{shift}\left(\Delta \mathrm{f}_{\mathrm{o}}\right)$.

$(529.8 \mathrm{MHz})$ while, the sensitivity of the nested metamaterial $(1.09 \mathrm{kHz} / \mathrm{kgf})$ was increased with respect to the classical metamaterial $(0.72 \mathrm{kHz} / \mathrm{kgf})$. Nested metamaterial structures are promising structures for adjusting the operating frequency and can be used in different applications other than monitoring fracture healing.

\section{REFERENCES}

[1] R. Melik, E. Unal, N. K. Perkgoz, C. Puttlitz, and H.V. Demir, "Wireless Metamaterial RF-MEMS Strain Sensors," Applied Physics Letters, vol. 95, 011106, (2009).

[2] R. Melik, E. Unal, N. K. Perkgoz, B. Santoni, D. Kamstock, C.M. Puttlitz, and H. V. Demir, "Nested Metamaterials for Wireless Strain Sensing", IEEE Journal of Selected Topics in Quantum Electronics, vol. 16 , pp. 450-458, (2010).

[3] D. R. Smith, "The reality of negative refraction", Physics World, vol. 16, pp. 23, 2003.

[4] D. Schurig, J. J. Mock, B. J. Justice, S. A. Cummer, J. B. Pendry, A. F. Starr, D. R. Smith, "Metamaterial Electromagnetic Cloak at Microwave Frequencies" Science, vol. 314, 977 (2006).

[5] D. R. Smith, "How to build a superlens", Science, vol. 308, pp. 502-503, 2005.

[6] JB Pendry, and SA Ramakrishna, "Focusing light using negative refraction", J. Phys. [Condensed Matter], vol. 15, pp. 6345-6364, 2003. 